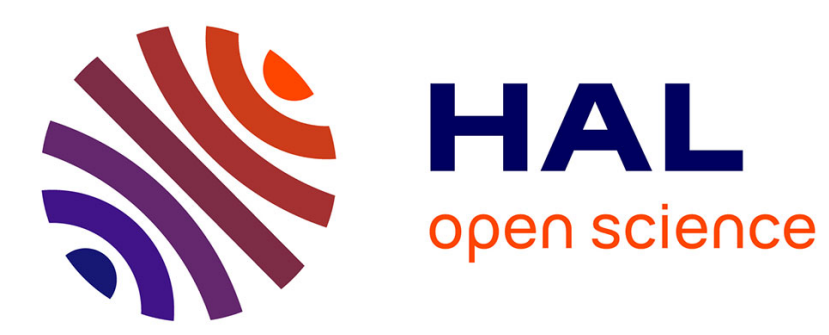

\title{
Lamination duale à un arbre réel
}

Arnaud Hilion

\section{- To cite this version:}

Arnaud Hilion. Lamination duale à un arbre réel. Séminaire de Théorie Spectrale et Géométrie, 2005, 24, pp.9-21. 10.5802/tsg.237 . hal-01218377

\section{HAL Id: hal-01218377 https://hal.science/hal-01218377}

Submitted on 21 Oct 2015

HAL is a multi-disciplinary open access archive for the deposit and dissemination of scientific research documents, whether they are published or not. The documents may come from teaching and research institutions in France or abroad, or from public or private research centers.
L'archive ouverte pluridisciplinaire HAL, est destinée au dépôt et à la diffusion de documents scientifiques de niveau recherche, publiés ou non, émanant des établissements d'enseignement et de recherche français ou étrangers, des laboratoires publics ou privés. 


\title{
Lamination duale à un arbre réel
}

\author{
Arnaud Hilion
}

5 avril 2007

\begin{abstract}
Résumé
Nous présentons des résultats reliant un arbre réel muni d'une action par isométries du groupe libre, sa lamination duale et les courants portés par cette dernière.
\end{abstract}

\section{Introduction}

Ce texte s'appuie sur un travail commun avec Thierry Coulbois et Martin Lustig [7, 8, 5, 6]. La motivation de ce travail est comprendre dans quelle mesure, la très jolie dualité entre les laminations géodésiques mesurées sur une surface hyperbolique d'une part, et les actions par isométries, petites, du groupe fondamental de cette surface sur des arbres réels d'autre part, peut (ou ne peut pas) être généralisée aux actions par isométries d'un groupe libre sur un arbre réel. Signalons le survol de Martin Lustig [15] qui traite du même sujet, partage beaucoup avec ce texte, et présente en outre une liste de questions ouvertes.

La section 1 rappelle brièvement les définitions du groupe libre, d'un arbre réel et de l'Outre-espace. On explique ensuite dans la section 2 la construction classique de l'arbre dual à une lamination géodésique mesurée sur une surface à bord. La section 3 introduit les laminations algébriques et les courants algébriques. Ces objets sont "intrinsèques" dans le sens qu'ils sont définis sans faire mention d'une base du groupe libre $F_{N}$. Si l'on se donne une base, on peut définir des objets équivalents : les laminations et les courants symboliques (qui sont de nature "dynamique symbolique") d'une part, les langages laminaires et les fonctions de Kolgomorov (qui sont plutôt d'inspiration "combinatoire") d'autre part. La définition de la lamination duale à un arbre réel est donnée dans section 4. Enfin, la section 5 est consacrée à énoncer quelques résultats liant un arbre et sa lamination duale.

\section{Outre-espace}

\subsection{Le groupe libre et son bord}

On note $F_{N}$ le groupe libre de rang $N \geqslant 2$. Une base $\mathcal{A}$ étant fixée, on peut voir $F_{N}=F(\mathcal{A})$ comme l'ensemble des mots (finis) $w=x_{1} \ldots x_{k}\left(x_{i} \in \mathcal{A} \cup \mathcal{A}^{-1}\right)$ 
réduits (i.e. $x_{i} \neq x_{i+1}^{-1}$ ) sur l'alphabet $\mathcal{A} \cup \mathcal{A}^{-1}$ (l'élément neutre de $F_{N}$ étant représenté par le mot vide 1$)$. La loi du groupe est la concaténation-réduction.

Le bord (de Gromov) $\partial F_{N}$ de $F_{N}$ peut être vu comme l'ensemble $\partial F(\mathcal{A})$ des mots $X=x_{1} \ldots x_{k} \ldots$ infinis à droite, réduits sur l'alphabet $\mathcal{A} \cup \mathcal{A}^{-1}$. On munit $\mathcal{A} \cup \mathcal{A}^{-1}$ de la topologie discrète et $\left(\mathcal{A} \cup \mathcal{A}^{-1}\right)^{\mathbb{N}^{*}}$ de la topologie produit. Le bord $\partial F_{N} \subset\left(\mathcal{A} \cup \mathcal{A}^{-1}\right)^{\mathbb{N}^{*}}$ hérite de la topologie induite : c'est un ensemble de Cantor. Le groupe libre $F_{N}$ agit (continûment) sur son bord par translations à gauche : si $w=x_{1} \ldots x_{k} \in F(\mathcal{A}), Y=y_{1} \ldots y_{l} \ldots \in \partial F(\mathcal{A})$, alors $w Y=x_{1} \ldots x_{k-i} y_{i+1} \ldots y_{l} \ldots \in \partial F(\mathcal{A})$ où $y_{1} \ldots y_{i}=x_{k}^{-1} \ldots x_{k-i+1}^{-1}$ est le plus long préfixe commun à $w^{-1}$ et $Y$.

Remarque 1. Le groupe libre $F_{N}$ et son bord $\partial F_{N}$ peuvent être définis sans faire mention d'une base $\mathcal{A}$. Par exemple : $F_{N}$ est le groupe fondamental d'un graphe de caractéristique d'Euler $N-1$; le bord $\partial F_{N}$ est le bord du revêtement universel (qui est un arbre) de ce graphe. L'utilisation d'une base se révèle être pratique pour définir (et plus encore étudier) des objets sur le groupe libre. Mais se pose alors le problème de savoir si ces objets sont "intrinsèques", i.e. ne dépendent pas du choix de la base. Ce sera le cas, dans la suite de ce texte, pour les laminations algébriques et les courants.

\subsection{Arbres réels}

Un arbre réel $T$ est un espace métrique non vide tel que :

- pour tous points $x, y \in T$, il existe un unique arc topologique (i.e. l'image dans $T$ d'un plongement d'un intervalle réel) dans $T$ joignant $x$ et $y$,

- cet arc est isométrique au segment réel $[0, d(x, y)]$,

où $d$ désigne la distance sur $T$. De manière équivalente, un arbre réel $T$ est un espace métrique, non vide, géodésique et 0-hyperbolique (au sens de Gromov).

\subsection{Action du groupe libre sur un arbre réel}

Soit $T$ un arbre réel. Considérons une isométrie $w$ de $T$. On définit la longueur de translation de $w$ dans $T$ par :

$$
\|w\|_{T}=\inf \{d(x, w x) \mid x \in T\} .
$$

Seuls deux cas sont possibles :

Cas 1: $\|w\|_{T}=0$. Dans ce cas, $w$ a un point fixe dans $T$, et l'on dit que $w$ est elliptique.

Cas 2: $\|w\|_{T}>0$. Dans ce cas, $w$ laisse un axe (i.e. l'image d'un plongement de $\mathbb{R}$ dans $T)$, noté $\operatorname{Axe}(w)$, invariant, et agit par translation de longueur $\|w\|_{T}$ sur $\operatorname{Axe}(w)$. On dit que $w$ est hyperbolique. dite :

On suppose que le groupe libre $F_{N}$ agit par isométries sur $T$. L'action est

- non triviale s'il n'existe pas de point de $T$ laissé fixe par $F_{N}$, 
- minimale s'il n'y a pas de sous arbre propre $F_{N}$-invariant,

- très petite si :

i) le stabilisateur d'un arc non dégénéré (i.e. non réduit à un point) de $T$ est un sous-groupe cyclique ou trivial de $F_{N}$

ii) un élément non trivial de $F_{N}$ ne fixe pas un tripode non dégénéré de $T$ (un tripode est l'enveloppe convexe dans $T$ de 3 points de $T$, il est non dégénéré s'il possède un point dont le complémentaire a 3 composantes connexes),

iii) un élément non trivial de $F_{N}$ ne renverse pas (i.e. ne permute pas les extémités d') un arc non dégénéré de $T$.

Une action de $F_{N}$ sur $T$ définit une fonction longueur $\|\cdot\|_{T}$ dans l'espace $\mathbb{R}^{F_{N}}$ des fonctions de $F_{N}$ dans $\mathbb{R}$, et donc aussi un point de l'espace projectif $\mathbb{P R}^{F_{N}}$.

Fait 1. Un arbre $T$ muni d'une action par isométries de $F_{N}$ non triviale, minimale et très petite est caractérisé (à isométrie $F_{N}$-équivariante près) par sa fonction longueur $\|\cdot\|_{T}$.

On désigne par $\overline{\mathrm{cv}}_{N}$ l'ensemble des arbres réels $T$ munis d'une action par isométries de $F_{N}$, minimale, non triviale, très petite. L'ensemble $\overline{\mathrm{cv}}_{N}$ s'injecte dans $\mathbb{R}^{F_{N}}$ via les fonctions longueurs : il hérite ainsi de la topologie induite.

On désigne par $\mathrm{cv}_{N} \subset \overline{\mathrm{cv}}_{N}$ le sous ensemble des actions libres et simpliciales, et on pose $\partial \mathrm{cv}_{N}=\overline{\mathrm{cv}}_{N} \backslash \mathrm{cv}_{N}$. C'est pour les éléments de $\partial \mathrm{cv}_{N}$ que l'on définira dans la section 4 une lamination duale.

Enfin, on désigne par $\mathrm{CV}_{N}$ (resp. $\overline{\mathrm{CV}}_{N}$ et $\partial \mathrm{CV}_{N}$ ) l'espace des classes d'homothétie d'éléments de $\mathrm{cv}_{N}$ (resp. $\overline{\mathrm{cv}}_{N}$ et $\left.\partial \mathrm{cv}_{N}\right): \mathrm{CV}_{N}$ est l'espace de Culler-Vogtmann ou Outre-espace. On pourra consulter [18] pour plus de détails.

Par la suite, on s'intéressera plus particulièrement aux arbres à orbites denses : on dit que $T$ est à orbites denses s'il existe un point de $T$ dont l'orbite (sous l'action de $F_{N}$ ) est dense dant $T$. Dans ce cas, toutes les orbites sont denses, si de plus l'action est très petite, on montre que le stabilisateur d'un arc non dégénéré est trivial.

Exemple 1. (i) Considérons un graphe de Cayley $\Gamma\left(F_{N}, \mathcal{A}\right)$ de $F_{N}$ relatif à une base $\mathcal{A}$, muni de l'action naturelle de $F_{N}$. On peut imposer que chaque arête soit isométrique à un segment réel non dégénéré, et ce de manière $F_{N}$-équivariante. On obtient un abre 'eel, simplicial, muni d'une action libre de $F_{N}$ par isométries.

(ii) Un exemple d'arbre réel non simplicial muni d'une action par isométries de $F_{N}$ est donné par l'arbre dual à une lamination géodésique mesurée sur une surface à bord. Cette construction est détaillée dans la section suivante.

Pour plus d'informations sur les arbres réels, on pourra consulter par exemple [1], [16] et [3]. 


\section{Chez les surfaces}

\subsection{Laminations géodésiques mesurées sur les surfaces}

Soit $S$ une surface orientable, compacte, de genre $g \geqslant 1$, à bord non vide. Son groupe fondamental $\pi_{1} S$ est un groupe libre $F_{N}$ de rang $N \geqslant 2$. On munit $S$ d'une structure hyperbolique telle que le bord de $S$ soit une réunion de géodésiques fermées. Le revêtement universel $\widetilde{S}$ de $S$ est un sous-ensemble convexe de l'espace hyperbolique $\mathbb{H}^{2}$, et $\pi_{1} S=F_{N}$ agit par isométries sur $\widetilde{S}$. Le bord de Gromov $\partial F_{N}$ de $F_{N}$ se plonge alors naturellement dans le bord de $\partial \mathbb{H}^{2}=S_{\infty}^{1}$ de $\mathbb{H}^{2}$.

Considérons une famille non vide de géodésiques de $\widetilde{S}$, appelées feuilles, deux à deux disjointes, telles que leur réunion $\widetilde{\mathfrak{L}}$ soit un fermé $F_{N}$-invariant de $\mathbb{H}^{2}$. La projection $\mathfrak{L}$ de $\widetilde{\mathfrak{L}}$ dans $S$ est appelée une lamination géodésique (voir [2]). La projection d'une feuille de $\widetilde{\mathfrak{L}}$ est une feuille de $\mathfrak{L}$.

Une lamination géodésique $\mathfrak{L}$ est dite minimale si $\mathfrak{L}$ est l'adhérence dans $S$ de chacune de ses feuilles. On dit que $\mathfrak{L}$ remplit $S$ si toute géodésique (sauf les composantes de bord) de $S$ rencontre $\mathfrak{L}$.

Soit $\mathcal{J}$ l'ensemble des arc lisses de $S$ dont les extrémités sont dans $S \backslash \mathfrak{L}$ et qui intersectent les feuilles de $\mathfrak{L}$ transversalement. Une mesure transverse sur $\mathfrak{L}$ est une application $\mathfrak{m}: \mathcal{J} \rightarrow \mathbb{R}^{+}$telle que (voir [12]) :

- la restriction $\mathfrak{m}_{\mid \gamma}$ de $\mathfrak{m}$ à tout $\gamma \in \mathcal{J}$ est une mesure de Borel $\sigma$-additive,

- $\mathfrak{m}(\gamma)=\mathfrak{m}\left(\gamma^{\prime}\right)$ s'il existe une isotopie entre $\gamma, \gamma^{\prime} \in \mathcal{J}$ réalisée par des éléments de $\mathcal{J}$,

$-\operatorname{si} \mathfrak{m}(\gamma)>0$, alors $\gamma \cap \mathfrak{L} \neq \emptyset$.

Une telle mesure transverse se relève naturellement à $\widetilde{S}$ en une mesure transverse $\widetilde{\mathfrak{m}}$ à $\widetilde{\mathfrak{L}}, F_{N}$-invariante.

\subsection{L'arbre réel dual à une lamination géodésique mesurée}

On définit une pseudo-distance $d_{\mathfrak{m}}$ sur $\widetilde{S}$ par :

- $d_{\mathfrak{m}}(x, y)=0$, si $x, y$ sont dans une même feuille de $\widetilde{\mathfrak{L}}$,

$-d_{\mathfrak{m}}(x, y)=\inf \{\widetilde{\mathfrak{m}}(\gamma): \gamma \in \widetilde{\mathcal{J}}$ joignant $x$ et $y\}$, sinon,

où $\widetilde{\mathcal{J}}$ est l'ensemble des $\operatorname{arcs}$ de $\widetilde{S}$ se projettant sur des arcs de $\mathcal{J}$.

On désigne par $T_{\mathfrak{m}}$ l'espace métrique obtenu en quotientant $\widetilde{S}$ par la relation d'équivalence $x \sim y$ si, et seulement si, $d_{\mathfrak{m}}(x, y)=0$. On note encore $d_{\mathfrak{m}}$ la distance sur $T_{\mathfrak{m}}$.

L'action de $F_{N}$ sur $\widetilde{S}$ induit une action par isométries sur $T_{\mathfrak{m}}$.

Fait 2. $T_{\mathfrak{m}}$ est un arbre réel, muni d'une action très petite de $F_{N}$.

A partir de l'action de $F_{N}$ sur $T_{\mathfrak{m}}$, on peut reconstruire la surface $S$ et la lamination géodésique mesurée dont $T_{\mathfrak{m}}$ est issu, en utilisant la machinerie de Rips (voir par exemple [1] ou [12]). 


\section{Laminations algébriques et courants}

\subsection{Le point de vue "intrinsèque"}

Le bord double de $F_{N}$ est défini comme :

$$
\partial^{2} F_{N}=\partial F_{N} \times \partial F_{N} \backslash \Delta,
$$

où $\Delta=\left\{(X, X) \mid X \in \partial F_{N}\right\}$. Le bord double est naturellement muni :

- de la topologie induite par celle de $\partial F_{N}$,

- de l'action de $F_{N}$ induite par l'action diagonale de $F_{N}$ sur $\partial F_{N} \times \partial F_{N}$,

- de l'involution $(X, Y) \mapsto(Y, X)$, appelée "flip".

Une lamination algébrique est un sous-ensemble non vide $L^{2} \subset \partial^{2} F_{N}$ qui est

- fermé,

- $F_{N}$-invariant,

- flip-invariant.

L'ensemble des laminations algébriques est noté $\Lambda^{2}\left(F_{N}\right)$.

Revenons au cas d'une lamination géodésique $\mathfrak{L}$ sur une surface (à bord) $S$.

Fait 3. Une lamination géodésique $\mathfrak{L} \subset S$ détermines une lamination algébrique $L^{2}(\mathfrak{L})$ qui est donnée par les couples de points terminaux des feuilles de $\tilde{\mathfrak{L}}$. On $a:$

$$
\mathfrak{L}=\mathfrak{L}^{\prime} \Longleftrightarrow L^{2}(\mathfrak{L})=L^{2}\left(\mathfrak{L}^{\prime}\right)
$$

Remarque 2. Le plongement de $\partial F_{N}$ dans $\partial \mathbb{H}^{2}=S_{\infty}^{1}$ induit un ordre cyclique $F_{N}$-invariant sur $\partial F_{N}$ : étant donnés quatre points $X_{1}, X_{2}, Y_{1}, Y_{2} \in \partial F_{N}$, l'ordre dans lequel on les lit en parcourant $S_{\infty}^{1}$ traduit le fait que les géodésiques de $\widetilde{S}$ joignant $X_{1}$ et $X_{2}$ d'une part, et $Y_{1}$ et $Y_{2}$ d'autre part, se coupent ou non.

Les feuilles d'une lamination géodésique $\mathfrak{L}$ étant deux à deux disjointes, la lamination algébrique $L^{2}(\mathfrak{L})$ a la propriété remarquable de "préserver l'ordre cyclique" sur $\partial F_{N}$ induit par le plongement dans $\partial \mathbb{H}^{2}=S_{\infty}^{1}$. Les laminations algébriques préservant un ordre cyclique sur $\partial F_{N}$ forment un sous-ensemble strict remarquable de $\Lambda^{2}\left(F_{N}\right)$.

Un courant $\mu$ est une mesure de Radon $\mu>0$ sur $\partial^{2} F_{N}, F_{N}$-invariante et flip-invariante. Le support $\operatorname{Supp}(\mu)$ est l'intersection des fermés de $\partial^{2} F_{N}$ sur le complémentaire desquels $\mu=0$. Il est clair que :

Fait 4. Le support d'un courant est une lamination algébrique.

Revenons au cas d'une lamination géodésique mesurée $(\mathfrak{L}, \mathfrak{m})$ sur une surface (à bord) $S$.

Fait 5. 1) Une mesure transverse $\mathfrak{m}$ sur $\mathfrak{L}$ détermines un courant $\mu_{\mathfrak{m}}$. On a :

$$
\mathfrak{m}=\mathfrak{m}^{\prime} \Longleftrightarrow \mu_{\mathfrak{m}}=\mu_{\mathfrak{m}^{\prime}}
$$

2) $S i(\mathfrak{L}, \mathfrak{m})$ est une lamination géodésique mesurée sur $S$, on a $\operatorname{Supp}\left(\mu_{\mathfrak{m}}\right) \subset$ $L^{2}(\mathfrak{L})$. Si de plus $\mathfrak{L}$ est minimale, alors $\operatorname{Supp}\left(\mu_{\mathfrak{m}}\right)=L^{2}(\mathfrak{L})$. 


\subsection{Laminations symboliques et courants symboliques}

Fixons une base $\mathcal{A}$ du groupe libre $F_{N}=F(\mathcal{A})$. Rappelons qu'un point du bord $X \in \partial F_{N}$ s'écrit comme un mot réduit infini $X_{\mathcal{A}}=x_{1} x_{2} x_{3} \ldots \in \partial F(\mathcal{A})$ in $\mathcal{A} \cup \mathcal{A}^{-1}$.

On désigne par $\Sigma_{\mathcal{A}}$ l'ensemble des mots bi-infinis réduits

$$
Z=\ldots z_{-i-1} z_{-i} \ldots z_{-1} z_{0} \cdot z_{1} z_{2} \ldots z_{i} z_{i+1} \ldots
$$

avec $z_{i} \in \mathcal{A} \cup \mathcal{A}^{-1}$ (comme précédemment, par "réduit", on entend que pour tout $\left.i \in \mathbb{Z}, z_{i} \neq z_{i+1}^{-1}\right)$. On munit $\mathcal{A} \cup \mathcal{A}^{-1}$ de la topologie discrète et $\left(\mathcal{A} \cup \mathcal{A}^{-1}\right)^{\mathbb{Z}}$ de la topologie produit : $\Sigma_{\mathcal{A}} \subset\left(\mathcal{A} \cup \mathcal{A}^{-1}\right)^{\mathbb{Z}}$ hérite de la topologie induite.

L'inverse $Z^{-1}$ d'un élément $Z=\ldots z_{i-1} z_{i} z_{i+1} \cdots \in \Sigma_{\mathcal{A}}$ est $Z^{-1}=$ $\ldots z_{i-1}^{\prime} z_{i}^{\prime} z_{i+1}^{\prime} \ldots$ où $z_{i}^{\prime}=z_{-i+1}^{-1}$ pour tout $i \in \mathbb{Z}$. Le décalage $\sigma: \Sigma_{\mathcal{A}} \rightarrow \Sigma_{\mathcal{A}}$ est défini par $\sigma(Z)=Z^{\prime}$ où $Z=\ldots z_{i-1} z_{i} z_{i+1} \ldots, Z^{\prime}=\ldots z_{i-1}^{\prime} z_{i}^{\prime} z_{i+1}^{\prime} \ldots$ et $z_{i}^{\prime}=z_{i+1}$ pour tout $i \in \mathbb{Z}$. Le décalage et l'inversion sont des homéomorphismes de $\Sigma_{\mathcal{A}}$.

Une lamination symbolique $L_{\mathcal{A}}$ dans la base $\mathcal{A}$ est un sous ensemble non vide de $\Sigma_{\mathcal{A}}$ qui est :

- fermé,

- invariant sous le décalage,

- symétrique (i.e. invariant sous l'inversion).

L'ensemble des laminations symboliques est noté $\Lambda_{\mathcal{A}}$. Les mots bi-infinis $Z \in L_{\mathcal{A}}$ sont appelés feuilles de $L_{\mathcal{A}}$.

Soit $(X, Y) \in \partial^{2} F_{N}$, et $X_{\mathcal{A}}, Y_{\mathcal{A}} \in \partial F(\mathcal{A})$ les mots infinis réduits correspondants. On obtient un mot bi-infini réduit $Z_{\mathcal{A}}$ en concaténant et réduisant $X_{\mathcal{A}}^{-1}$ et $Y_{\mathcal{A}}$ :

$$
Z_{\mathcal{A}}=X_{\mathcal{A}}^{-1} Y_{\mathcal{A}}=\ldots x_{i+2}^{-1} x_{i+1}^{-1} x_{i}^{-1} \cdot y_{i} y_{i+1} y_{i+2} \ldots
$$

où $x_{1} \ldots x_{i-1}=y_{1} \ldots y_{i-1}$ est le plus long préfixe commun à $X_{\mathcal{A}}$ et $Y_{\mathcal{A}}$. Considérons une lamination algébrique $L^{2} \subset \partial^{2} F_{N}$.

Fait $6([7])$. L'ensemble des mots bi-infinis obtenus par le procédé précédant à partir de toutes les feuilles $(X, Y)$ d'une lamination algébrique $L^{2}$ forme une lamination symbolique $L_{\mathcal{A}}\left(L^{2}\right)$. On obtient ainsi une bijection entre $\Lambda^{2}\left(F_{N}\right)$ et $\Lambda_{\mathcal{A}}$.

Un courant symbolique est une mesure de Borel $\sigma$-invariante et invariante par l'inversion. Le support d'un courant symbolique est une lamination symbolique. Un courant algébrique détermine un unique courant symbolique.

\subsection{Langages laminaires et fonctions de Kolgomorov}

Le langage laminaire de la lamination algébrique $L^{2}$, ou de la lamination symbolique $L_{\mathcal{A}}\left(L^{2}\right)$, est l'ensemble $\mathcal{L}_{\mathcal{A}}\left(L^{2}\right) \subset F(\mathcal{A})$ de tous les sous-mots finis $w$ des feuilles de $L_{\mathcal{A}}\left(L^{2}\right)$. 
Réciproquement, un ensemble infini $\mathcal{L}_{\mathcal{A}} \subset F(\mathcal{A})$ définit une lamination algébrique $L^{2}\left(\mathcal{L}_{\mathcal{A}}\right)$ dont les feuilles sont les couples $(X, Y)$ tels que tout sous mot fini $w \in F(\mathcal{A})$ de $X_{\mathcal{A}}^{-1} Y_{\mathcal{A}}$, ou son inverse $w^{-1}$, est sous mot d'un mot de $\mathcal{L}_{\mathcal{A}}$.

On obtient ainsi (voir [7]) une bijection canonique entre l'ensemble des laminations algébriques $\Lambda^{2}\left(F_{N}\right)$ et les langages laminaires $\Lambda_{\mathcal{L}}$.

Dans le contexte des langages laminaires, on peut définir l'équivalent d'un courant de la manière suivante. Une fonction de Kolmogorov est une application

$$
\mu_{\mathcal{A}}: F(\mathcal{A}) \longrightarrow \mathbb{R}^{+}
$$

qui vérifie, pour tout mot réduit $w=y_{1} \ldots y_{k} \in F(\mathcal{A})$ :

(i) $\mu_{\mathcal{A}}\left(w^{-1}\right)=\mu_{\mathcal{A}}(w)$

(ii) $\mu_{\mathcal{A}}(w)=\sum_{y \neq y_{k}^{-1}} \mu_{\mathcal{A}}(w y)=\sum_{y \neq y_{1}^{-1}} \mu_{\mathcal{A}}(y w) \quad\left(\right.$ pour $\left.y \in \mathcal{A} \cup \mathcal{A}^{-1}\right)$.

Un courant $\mu$ définit une fonction de Kolgomorov, et réciproquement. Le courant $\mu$ correspond à la fonction de Kolmogorov $\mu_{\mathcal{A}}$ si, et seulement si, pour tout mot réduit $w=y_{1} \ldots y_{k} \in F(\mathcal{A})$ on a

$$
\mu_{\mathcal{A}}(w)=\mu\left(C_{\mathcal{A}}^{2}(w)\right),
$$

où $C_{\mathcal{A}}^{2}(w) \subset \partial^{2} F(\mathcal{A})=\partial^{2} F_{N}$ est le cylindre algébrique définit par :

$$
\left\{\left(X_{\mathcal{A}}, w X_{\mathcal{A}}^{\prime}\right) \mid X_{\mathcal{A}}=x_{1} x_{2} \ldots, X_{\mathcal{A}}^{\prime}=x_{1}^{\prime} x_{2}^{\prime} \ldots \in \partial F(\mathcal{A}), x_{1} \neq y_{1}, x_{1}^{\prime} \neq y_{k}^{-1}\right\} .
$$

Pour plus de détails, voir [10] ou [11].

Remarque 3. Dans [7], on définit trois topologies (métrisables) naturelles sur l'ensemble des laminations algébriques $\Lambda^{2}\left(F_{N}\right)$, sur l'ensemble des laminations symboliques $\Lambda_{\mathcal{A}}$, et sur l'ensemble des langages laminaires $\Lambda_{\mathcal{L}}$. On décrit aussi l'action du groupe des automorphismes extérieurs $\operatorname{Out}\left(F_{N}\right)$ de $F_{N}$ sur ces trois espaces. On montre que les bijections entre $\Lambda^{2}\left(F_{N}\right), \Lambda_{\mathcal{A}}$ et $\Lambda_{\mathcal{L}}$ explicitées plus haut, sont en fait des homéomorphismes $\operatorname{Out}\left(F_{N}\right)$-équivariants.

\section{Lamination algébrique duale à une arbre réel}

Soit $T$ un arbre réel muni d'une action par isométries de $F_{N}$. On suppose comme précédemment que l'action est non triviale, minimale et très petite, mais on exclut le cas d'une action libre sur un arbre $T$ simplicial (i.e. $T \in \partial \mathrm{cv}_{N}$ ).

Fixons une base $\mathcal{A}$ de $F_{N}$. On définit $\mathcal{L}_{\mathcal{A}}(T) \subset F(\mathcal{A})$ comme l'ensemble des mots réduits $w \in F(\mathcal{A})$ tels que, pour tout $\varepsilon>0$, il existe un mot $v \in F(\mathcal{A})$ cycliquement réduit dont $w$ est un sous-mot et dont la distance de translation dans $T$ vérifie $\|v\|_{T}<\varepsilon$.

La lamination algébrique duale à $T$, notée $L^{2}(T)$, est définie comme la lamination algébrique dont le langage laminaire est $\mathcal{L}_{\mathcal{A}}(T)$.

Fait 7. Cette définition est en fait indépendante du choix de la base $\mathcal{A}$ (cf [8]). 
Remarque 4. Dans le cas d'une action libre simpliciale, la définition reste valable, mais $L^{2}(T)$ est l'ensemble vide (et donc n'est pas une lamination algébrique).

Considérons le cas où $T=T_{\mathfrak{m}}$ est l'arbre dual d'une lamination géodésique mesurée $(\mathfrak{L}, \mathfrak{m})$ sur une surface $S$.

Fait 8. On $a$ :

$$
L^{2}(\mathfrak{L}) \subset L^{2}\left(T_{\mathfrak{m}}\right) .
$$

Si de plus $\mathfrak{L}$ est minimale et remplit $S$, alors la lamination $L^{2}\left(T_{\mathfrak{m}}\right)$ s'obtient en rajoutant à la lamination $L^{2}(\mathfrak{L})$ ses feuilles diagonales.

Considérons une lamination géodésique $\mathfrak{L}$ minimale et qui remplit $S$. Les composantes connexes du complémentaire de $\widetilde{\mathfrak{L}}$ dans $\widetilde{S}$ sont des $n$-gones idéaux (cf [2]). Lorsque $n \geqslant 4$, il y a des géodésiques dans le $n$-gone joignant deux sommets du $n$-gone : ce sont les "feuilles diagonales" de $\widetilde{\mathfrak{L}}$.

\section{Relations entre un arbre et sa lamination duale}

\subsection{La topologie métrique sur $\widehat{T}$}

Considérons un arbre réel $T$. Un rayon est l'image d'un plongement de $\mathbb{R}$ dans $T$ Deux rayons de $T$ sont équivalents s'ils ont une intersection non compacte. Le bord de Gromov de $T$, que l'on note $\partial T$, est l'ensemble des classes d'équivalence de rayons dans $T$. On désigne par $\bar{T}$ le complété (de Cauchy) de $T$ et par $\widehat{T}$ la réunion de $\bar{T}$ et $\partial T$.

Soit $P$ un point de $T$. Une composante connexe de $T \backslash\{P\}$ est appelée une direction de $T$ en $P$.

La distance sur $T$ s'étend canoniquement en une distance sur $\bar{T}$, et induit canoniquement une topologie sur $\widehat{T}$, appelée topologie métrique :

- une base de voisinages d'un point de $\bar{T}$ est donnée par les boules ouvertes centrées en ce point,

- une base de voisinages d'un point $X \in \partial T$ est donnée par l'ensemble des directions de $T$ qui ont une intersection non bornée avec un rayon représentant $X$.

\subsection{La topologie des observateurs sur $\widehat{T}$}

On peut généraliser la définition d'une direction : si $P$ un point de $\widehat{T}$, on appelle direction de $\widehat{T}$ en $P$ toute composante connexe de $\widehat{T} \backslash\{P\}$. La topologie des observateurs sur $\widehat{T}$ est la topologie engendrée par les directions de $\widehat{T}$. On désigne par $\widehat{T}^{\text {obs }}$ l'espace $\widehat{T}$ muni de la topologie des observateurs.

Proposition 9 ([6]). Soit $T$ un arbre réel. Alors :

- $\widehat{T}^{\text {obs }}$ est compact,

- l'identité $i d: \widehat{T} \rightarrow \widehat{T}^{\text {obs }}$ est continue, 
- la topologie métrique et la topologie des observateurs sont les mêmes sur les sous-arbres finis de T (un sous-arbre fini est l'enveloppe convexe d'un nombre fini de points de T).

Il est à noter que si $T$ est à orbites denses, alors ni $T$, ni $\widehat{T}$ ne sont localement compacts. Typiquement, considérons une suite $P_{k}$ de points de $\widehat{T}$ telle que :

- $d\left(P_{0}, P_{k}\right)$ ne tend pas vers 0

- $d\left(P_{0}, Q_{k}\right)$ tend vers 0 ,

où $Q_{k}$ est défini par $\left[P_{0}, Q_{k}\right]=\left[P_{0}, P_{k}\right] \cap\left[P_{0}, P_{k+1}\right]$. La suite $P_{k}$ ne converge pas dans $\widehat{T}$ muni de la topologie métrique, mais converge vers $P_{0}$ dans $\widehat{T}^{\text {obs }}$.

\subsection{L'application $\mathcal{Q}$}

On suppose par la suite que $T \in \overline{c v}_{N}$ est un arbre réel à orbites denses.

Levitt et Lustig construisent dans [14] une application

$$
\mathcal{Q}: \partial F_{N} \longrightarrow \widehat{T} \text {. }
$$

Cette application est $F_{N}$-équivariant et surjective, mais n'est, en général, ni injective ni continue.

Lorsque $T=T_{\mathfrak{m}}$ est l'arbre dual à une lamination géodésique mesurée $(\mathfrak{L}, \mathfrak{m})$ sur une surface $S$, l'application $\mathcal{Q}$ est donnée par l'application "d'inclusionrétraction" canonique $\partial F_{N} \subset \mathbb{H}^{2} \cup S_{\infty}^{1} \rightarrow \bar{T}_{\mathfrak{m}} \cup \partial T_{\mathfrak{m}}$. En particulier, si $X$ est un point de $S_{\infty}^{1}$ atteint par une feuille de $\mathfrak{L}$, alors $\mathcal{Q}(X)$ est précisément le point de $T$ défini par cette feuille.

Théorème 10 ([8]). Si $T \in \overline{c v}_{N}$ est à orbites denses, alors

$$
L^{2}(T)=\left\{(X, Y) \in \partial^{2} F_{N} \mid \mathcal{Q}(X)=\mathcal{Q}(Y)\right\} .
$$

On désigne par $\partial F_{N} / L^{2}(T)$ le quotient du bord $\partial F_{N}$ par la relation d'équivalence : $X \sim Y$ si, et seulement si, $(X, Y) \in L^{2}(T)$. D'après le théorème 10 , l'application $\mathcal{Q}$ induit une bijection $F_{N}$-équivariante de $\partial F_{N} / L^{2}(T)$ sur $\widehat{T}$.

Théorème $11([6])$. Soit $T \in \overline{c v}_{N}$ à orbites denses. L'application $F_{N}$-équivariante surjective $\mathcal{Q}: \partial F_{N} \rightarrow \widehat{T}^{\text {obs }}$ est continue. De plus, si l'on munit $\partial F_{N} / L^{2}(T)$ de la topologie quotient, l'application $\mathcal{Q}$ induit un homéomorphisme $F_{N}$-équivariant

$$
\partial F_{N} / L^{2}(T) \longrightarrow \widehat{T}^{o b s} \text {. }
$$

Fait 12. Le théorème 11 montre que $\widehat{T}^{\text {obs }}$ est entièrement déterminé par $L^{2}(T)$. Mais $T$ (ou $\widehat{T}$ ) n'est pas déterminé par $L^{2}(T)$, (cf [4]).

Par exemple, si $\mathfrak{L} \subset S$ est une lamination géodésique minimale, qui remplit $S$, et non uniquement ergodique (de telles laminations existent, cf [17, 13]), deux mesures ergodiques $\mathfrak{m}_{1}$ et $\mathfrak{m}_{2}$ projectivement distinctes sur $\mathfrak{L}$ donnent lieu à deux arbres duaux $T_{1}$ et $T_{2}$ dans $\overline{\mathrm{cv}}_{N}$, à orbites denses, qui ont la même lamination duale $L^{2}\left(T_{1}\right)=L^{2}\left(T_{2}\right)$ (i.e. $\widehat{T}_{1}^{\text {obs }}=\widehat{T}_{2}^{\text {obs }}$ ). Mais on montre dans [4] qu'il n'existe pas d'homéomorphisme $F_{N}$-équivariant entre $T_{1}$ et $T_{2}$. 


\subsection{La distance duale sur $T$}

Soit $T \in \overline{\mathrm{cv}}_{N}$ à orbites denses. Alors $\mathcal{Q}: \partial F_{N} \rightarrow \widehat{T}$ induit une application $F_{N}$-équivariante $\mathcal{Q}^{2}: L^{2}(T) \rightarrow \bar{T}$. Bien que l'application $\mathcal{Q}$ ne soit pas en général continue, on a :

Proposition $13([8])$. L'application $\mathcal{Q}^{2}: L^{2}(T) \rightarrow \bar{T}$ est continue.

Considérons un courant $\mu$ porté par $L^{2}(T): \operatorname{Supp}(\mu) \subset L^{2}(T)$. Puisque $\mathcal{Q}^{2}$ est continue, on peut transporter $\mu$ par $\mathcal{Q}^{2}$ : on obtient une mesure de Borel $F_{N}$-invariante $\mu_{\bar{T}}$ sur $\bar{T}$. On définit une "pseudo-distance" sur $\bar{T}$, en posant :

$$
d_{\mu}(x, y)=\mu_{\bar{T}}([x, y]) \quad\left(=\mu\left(\left(\mathcal{Q}^{2}\right)^{-1}([x, y])\right)\right)
$$

pour tout $x, y \in \bar{T}$. On parle de "pseudo-distance" car, a priori, deux points distincts peuvent être à distance $d_{\mu}$ nulle ou infinie.

Fait 14. Soit $(\mathfrak{L}, \mathfrak{m})$ une lamination géodésique mesurée sur une surface $S$. On désigne par $T_{\mathfrak{m}}$ l'arbre dual (et par $d_{\mathfrak{m}}$ la distance sur $T_{\mathfrak{m}}$ ). La mesure transverse $\mathfrak{m}$ définit un courant $\mu_{\mathfrak{m}}$ qui vérifie $\operatorname{Supp}\left(\mu_{\mathfrak{m}}\right) \subset L^{2}(\mathfrak{L}) \subset L^{2}\left(T_{\mathfrak{m}}\right)$. Dans ce cas, on $a$ :

$$
d_{\mu_{\mathfrak{m}}}=d_{\mathfrak{m}}
$$

Cette parfaite dualité n'a plus cours, en toute généralité, pour un arbre réel quelconque. Dans la section suivante, on explique comment contruire un contre-exemple.

\subsection{Un exemple d'arbre dont la distance duale diffère de la distance}

Un automorphisme $\varphi$ de $F_{N}$ est dit à puissances irréductibles (ou iwip) s'il n'existe pas de factor libre propre non-trivial de $F_{N}$ qui soit envoyé sur un conjugué de lui-même par une puissance (positive) de $\varphi$.

Fait 15. Si $\varphi \in \operatorname{Aut}\left(F_{N}\right)$ est iwip, alors il existe un unique (à homothétie près) arbre réel $T_{\varphi}$ muni d'une action très petite, laissé projectivement invariant, avec un facteur de dilatation $\lambda_{\varphi}>1$ : pour tout $w \in F_{N}$, on a

$$
\|\varphi(w)\|_{T_{\varphi}}=\lambda_{\varphi}\|w\|_{T_{\varphi}} .
$$

De plus $T_{\varphi}$ est à orbites denses.

Les automorphismes iwip de $F_{N}$ sont considérés comme les analogues, pour le groupe libre, des homémorphismes pseudo-Anosov d'une surface hyperbolique. Plus précisément, si $S$ est une surface possédant une seule composante de bord, alors un homéomorphisme pseudo-Anosov $f$ de $S$ induit un automorphisme $\varphi$ iwip sur $F_{N}=\pi_{1}(S)$. L'homéomorphisme $f$ fixe deux laminations géodésiques mesurées, la lamination stable $\left(\mathfrak{L}^{+}, \mathfrak{m}^{+}\right)$et la lamination instable $\left(\mathfrak{L}^{-}, \mathfrak{m}^{-}\right)$: il existe un facteur $\lambda_{f}>1$ tel que $f_{*} \mathfrak{m}^{+}=\lambda_{f}^{-1} \mathfrak{m}^{+}$et $f_{*} \mathfrak{m}^{-}=\lambda_{f} \mathfrak{m}^{-}$ (cf $[9,2])$. Dans ce cas $T_{\varphi}=T_{\mathfrak{m}^{-}}, T_{\varphi^{-1}}=T_{\mathfrak{m}^{+}}, \lambda_{\varphi}=\lambda_{\varphi^{-1}}=\lambda_{f}$. 
Fait 16. Il existe des automorphismes iwip $\varphi$ de $F_{N}$ tels que $\lambda_{\varphi} \neq \lambda_{\varphi^{1}}$.

C'est par exemple le cas de l'automorphisme de $F_{3}=\langle a, b, c\rangle$ défini par $\varphi(a)=a b, \varphi(b)=a c, \varphi(c)=a$.

Considérons un tel automorphisme $\varphi$. Cet automorphisme agit sur l'arbre réel $T_{\varphi}$ en multipliant la distance par un facteur $\lambda_{\varphi}$. D'autre part, la lamination duale $L^{2}\left(T_{\varphi}\right)$ est uniquement ergodique, i.e. porte, à multiplication par un scalaire non nul près, un seul courant $\mu$ (c'est un fait général pour tous les automorphismes iwip). On peut montrer que $\varphi$ agit sur $L^{2}\left(T_{\varphi}\right)$ en multipliant le courant $\mu$ par $\lambda_{\varphi^{-1}}$. Donc $\varphi$ agit sur $T_{\varphi}$ en multipliant la distance duale $d_{*}=d_{\mu}$ par $\lambda_{\varphi^{-1}}$. C'est la clé de la preuve du :

Théorème $17([5])$. Si $\varphi \in$ Aut $\left(F_{N}\right)$ est iwip avec $\lambda_{\varphi} \neq \lambda_{\varphi^{-1}}$, alors la pseudodistance duale $d_{*}$ est totalement dégénérée sur $T_{\varphi}$ : soit $d_{*}(x, y)=0$ pour tout $x, y \in T_{\varphi}$, soit $d_{*}(x, y)=\infty$ pour tout $x, y \in T_{\varphi}, x \neq y$.

\section{Références}

[1] M. Bestvina, $\mathbb{R}$-trees in topology, geometry, and group theory, Handbook of geometric topology, North-Holland, Amsterdam, 2002, pp. 55-91.

[2] A. Casson and S. Bleiler, Automorphisms of surfaces after Nielsen and Thurston, London Mathematical Society Student Texts, vol. 9, Cambridge University Press, Cambridge, 1988.

[3] M. Cohen and M. Lustig, Very small group actions on $\mathbf{R}$-trees and Dehn twist automorphisms, Topology 34 (1995), no. 3, 575-617.

[4] T. Coulbois, A. Hilion, G. Levitt, and M. Lustig, en préparation.

[5] T. Coulbois, A. Hilion, and M. Lustig, $\mathbb{R}$-trees and laminations for free groups III : Currents and dual $\mathbb{R}$-tree metrics, preprint.

[6] _ Non uniquely ergodic r-trees are topologically determined by their algebraic lamination, preprint available on http ://junon.u-3mrs.fr/hilion/, 2005.

[7] _, $\mathbb{R}$-trees and laminations for free groups I : Algebraic laminations, arXiv :math.GR/0609416, 2006.

[8], $\mathbb{R}$-trees and laminations for free groups II : The dual lamination of an $\mathbb{R}$-tree, arXiv :math.GR/0702281, 2007.

[9] A. Fathi, F. Laudenbach, and V. Poenaru (eds.), Travaux de Thurston sur les surfaces, Astérisque, vol. 66-67, Société Mathématique de France, Paris, 1976.

[10] I. Kapovich, Currents on free groups, ArXiv :math.GR/0311053, 2005.

[11] I. Kapovich and M. Lustig, The actions of Out $\left(F_{k}\right)$ on the boundary of outer space and on the space of currents : minimal sets and equivariant incompatibility, arXiv :math/0605548, 2006.

[12] Michael Kapovich, Hyperbolic manifolds and discrete groups, Progress in Mathematics, vol. 183, Birkhäuser Boston Inc., Boston, MA, 2001. 
[13] H. Keynes and D. Newton, A "minimal", non-uniquely ergodic interval exchange transformation, Math. Z. 148 (1976), no. 2, 101-105.

[14] G. Levitt and M. Lustig, Irreducible automorphisms of $F_{n}$ have north-south dynamics on compactified outer space, J. Inst. Math. Jussieu 2 (2003), 5972 .

[15] M. Lustig, $\mathbb{R}$-trees - currents - laminations : a delicate relationship, preprint, 2007.

[16] P. Shalen, Dendrology of groups : an introduction, Essays in group theory, Math. Sci. Res. Inst. Publ., vol. 8, Springer, New York, 1987, pp. 265-319.

[17] W. Veech, Strict ergodicity in zero dimensional dynamical systems and the Kronecker-Weyl theorem mod 2, Trans. Amer. Math. Soc. 140 (1969), 133.

[18] K. Vogtmann, Automorphisms of free groups and outer space, Proceedings of the Conference on Geometric and Combinatorial Group Theory, Part I (Haifa, 2000), vol. 94, 2002, pp. 1-31. 